\title{
Neuzeitliche Gletscherschwankungen
}

\section{Zum Begriff des Gletschervorfeldes}

Den meisten Lesern wird die unmittelbare Umgebung von Gletschern bestens vertraut sein. Dieses schuttreiche, noch wenig bis fast keine Vegetation tragende Areal, beidseitig von scharfkantigen Moränen begrenzt, hebt sich von den gut bewachsenen Talhängen und -böden deutlich ab. Durch den massiven Gletscherschwund, der nach einer längeren Zeit größerer Gletscherausdehnung ${ }^{1}\left(1600-1850^{2}\right)$ einsetzte, wurde hier ein Neuland freigelegt, das dem Glazialmorphologen wie auch dem Botaniker mannigfaltige Möglichkeiten für Forschungsarbeit eröffnet.

Über das Verhalten der Gletscher während der letzten 300-350 Jahre ist man recht gut im Bilde, existieren doch aus dieser Zeitspanne viele Bild- und Schriftquellen (vgl. LE ROY LADURIE 1967, zUMBüHL 1980). Mit der Gliederung der Moränen in der näheren Umgebung der heutigen Gletscher beschäftigte sich KINZL $(1929,1932)$ eingehend. Seine Untersuchungen in den Ost- und Westalpen ermöglichten ihm eine Unterteilung der Moränen nach ihrer Formfrische und nach dem Grad der Vegetationsbedeckung. So unterscheidet KINZL sogenannte "Fünfzigermoränen» (Vorstoß 1850) und «Zwanzigermoränen» (Vorstoß 1820) neben älteren Moränen, die er als «frührezent» bezeichnet und in zwei Hauptgruppen gliedert (1929, p. 103). Die erste Gruppe, die jüngeren «frührezenten» Moränen, nennt er Fernaumoränen. Die Stellung der älteren «frührezenten» Moränen, die weit außerhalb der Fernaumoränen liegen und deshalb einer viel größeren Gletscherausdehnung entsprechen, verweist KINZL in die enge Nachbarschaft der Daunmoränen (1929, p. 119) und bezeichnet diesen Gletscherstand als «Eggessen-Stadium» (Begriffe "Daun» und "Egesen" S. MAISCH 1981 und dieses Heft). Vom Begriff «frührezent» kommt KINZL in einer späteren Arbeit (KINZL 1932) jedoch ab und verwendet an dessen Stelle die Bezeichnung «nacheiszeitlich». Schon 1929 wies er auf die verschiedenen Auffassungen von «frührezent) in der damaligen Literatur hin (1929, p.69/70): Zum Beispiel braucht LUCERNA (1913, p. 327) diese Bezeichnung für die 1850er, 1820er als auch für die älteren Moränen, die diese Größenordnung erreicht haben; bei BüHLER $(1928$, p. 24) sind unter «frührezent» alle prähistorischen Gletscherstände bis hin zum Daunstadium enthalten, und die italienischen Autoren (z. B. NANGERONI 1927, p. 97ff.) verwenden dafür den Begriff "sottostadi dauni», der alle Nachdaunmoränen umfaßt.

Um die Frage nach den jüngeren "frührezenten» Moränen zu beantworten, stützt sich KINZL (1929, p. 114ff.) auf historisches Quellenmaterial aus dem 17. Jahrhundert. Er ordnet die Ablagerung dieser Moränen dem Gletschervorstoß zu, «der am Beginn des 17. Jahrhunderts einsetzt und während dieses Jahrhunderts, in den einzelnen Gebieten zu verschiedener Zeit, bei vielen Gletschern zur größten überhaupt bekannten Ausdehnung geführt hat». Als Beispiele nennt KINZL die Vorstöße der Grindelwaldgletscher um 1600, die Untersuchungen von MougIN (1912) an den Gletschern im Mont Blanc-Gebiet, die ebenfalls kräftig vorstießen, und weiter einen Hinweis von FINSTERWALDER (1897), daß der Vernagtferner seinen größten Stand um 1680 erreicht haben soll.

Basierend auf diesen Feststellungen, kommt KINZL zum Schluß, daß eine Reihe von Moränen, die den «Fernaumoränen» entsprechen, nachweislich aus dem 17. Jahrhundert stammen und somit einem der Vorstöße aus dieser Zeit zugeordnet werden können. Mit der früheren Annahme einer postglazialen Wärmezeit mit einer Erhöhung der Schneegrenze um 300-400 $\mathrm{m}$ und einem damit verbundenen weitgehenden Abschmelzen der Gletscher wuchs die Bedeutung der zu Beginn des 17. Jahrhunderts einsetzenden Gletschervorstoßphase, und dementsprechend hoch war ihr Stellenwert, den man ihr innerhalb der Nacheiszeit beimaß. Zudem war die Auffassung verbreitet, daß die heutigen Gletscher keine Relikte der letzten Eiszeit sind, sondern eine selbständige Vereisung darstellen (MATTHES 1939, 1940; CHARLESWORTH 1957).

Die gut erhaltenen Moore im extramoränen Bereich der jüngsten Moränen veranlaßten KINZL (1932, p. 360) denn auch zum Schluß, daß «tatsächlich die Moränen des 17. Jahrhunderts und die ihnen in der Größenordnung vergleichbaren Moränen des 19. Jahrhunderts

Hanspeter Holzhauser, dipl. Geograph,

Geographisches Institut der Universität Zürich

Postfach, 8033 Zürich 
die größten Gletscherstände nach der postglazialen Wärmezeit bezeichnen". Das Gletschervorfeld definierte er aufgrund dieser Auffassung wie folgt (1949, p. 82): «Unter Gletschervorfeld wird das Gelände zwischen dem heutigen Gletscherende und den Moränen des Vorstoßes von 1850 und der übrigen nachwärmezeitlichen Gletschervorstöße verstanden.»

Diese Definition des Gletschervorfeldes muß nach den heutigen Erkenntnissen präzisiert werden. Wie allgemein bekannt ist, wird das Ende des Spätglazials um 10200 Radiokarbonjahre vor heute (d. h. vor 1950) festgesetzt. Damals schmolzen die Gletscher auf heutige Größenordnung zurück. Wie mittels morphologischer Untersuchungen und ${ }^{14} \mathrm{C}$-Datierungen nachgewiesen werden konnte, bewegten sich die Gletscher in der darauffolgenden Zeit, dem Postglazial, nie mehr wesentlich über die Größenordnung von 1850 hinaus (vgl. GAMPER/SUTER, dieses Heft). Über diesen postglazialen Abschnitt schreibt PATZELT (1980, p. 15): «Er ist gekennzeichnet durch Gletscher- und Klimaschwankungen, die mit kleiner Amplitude um gegenwärtige Verhältnisse pendeln (...).» Mehrmals erreichten die Gletscher während des Postglazials Ausdehnungen der Größenordnung von 1850. Da folglich Moränen der letzten Hochstände mit älteren Moränen eng benachbart sein können, die schon vor einigen tausend Jahren abgelagert wurden, umfaßt das Gletschervorfeld im weiteren Sinne alle postglazialen Moränen. Dabei kann die das Vorfeld begrenzende Moräne je nach Gletscher verschieden alt sein, da nicht jeder Gletscher gleich reagierte.

Aus methodischen Erwägungen unterteilen wir das Postglazial in eine Vorneuzeit und in eine Neuzeit (FURRER et al. 1978). Im Bereich der Neuzeit, die wir in Anlehnung an die Historiker um 1500 beginnen lassen, berichten uns historische Dokumente von Gletscherbewegungen. Vor 1500 sind solche Dokumente äußerst spärlich vertreten, und wir müssen uns vorwiegend auf Feldbefunde und Radiokarbondatierungen abstützen, um eine Gletschergeschichte rekonstruieren zu können. Innerhalb der Neuzeit, die uns in diesem Artikel beschäftigen soll, sprechen wir folglich von neuzeitlichen Gletscherständen bzw. -ausdehnungen. Das Gletschervorfeld im engeren Sinne wird hier als Gelände zwischen den heutigen Gletscherumrissen und den Moränen der neuzeitlichen Hochstände (17.-19. Jahrhundert) verstanden.

Im folgenden kommen Hinweise zur Sprache, vorwiegend aus schriftlichem Quellenmaterial gewonnen, die Aussagen zur Aufhellung der Gletschergeschichte der vergangenen rund 400 Jahre erlauben. Eine Beschränkung auf einige wenige ausgewählte «Indizien» drängte sich in Anbetracht der Kürze dieses Artikels auf. Was die einzelnen Methoden betrifft, die zur Erforschung des Gletscherverhaltens während der Neuzeit herangezogen werden, so sei auf den Artikel von HOLZHAUSER/WETTER (1982) hingewiesen.

\section{Das Verhalten der Gletscher während der Neuzeit}

\subsection{Erste Hinweise}

Mit den ersten Landesbeschreibungen und -chroniken treten auch Beschreibungen der Alpen auf. Diese fallen noch sehr summarisch aus, denn die Eisregionen waren bei den damaligen Autoren aus eigener Anschauung meistens nicht bekannt. Man begnügte sich mit der Betrachtung dieser gefährlichen Gebiete aus der Ferne, und falls Reisen unternommen wurden, hielt man sich an die bequem begehbaren Hauptrouten und mied die abgelegenen Seitentäler.

Versuche, die Alpenregionen auch kartographisch zu erfassen, sind früh unternommen worden ${ }^{3}$. Diese kartographischen Zeugnisse sind aber noch sehr unpräzis, und nur wenige eignen sich für gletschergeschichtliche Untersuchungen. Iмноғ (1974, p. 131) schreibt darüber: "Doch selbst in unseren Regionen vermögen alte Karten, solche vor etwa 1850, die landschaftlichen Zustände nur ungenügend aufzuhellen, denn ihre geometrischen Gefüge entsprechen bloßen Skizzen; sie sind voller grober Verzerrungen oder Verzeichnungen und inhaltlich äußerst lückenhaft.»

Ähnlich verhält es sich auch mit schriftlichen Aufzeichnungen. Vor allem sind dies sehr weit und summarisch verfaßte Beschreibungen, besonders was die Betrachtung der Gletscher anbelangt. Die damaligen Gelehrten beschränkten sich hauptsächlich darauf, über die Beschaffenheit und die Nutzung der Gletscher bzw. des Gletschereises zu mutmaßen; wie dies z. B. bei MÜNSTER (1544) nachzulesen ist:

"Wiewol die gletscher nit bergwerck noch metallen seind/seind sie doch gleichformig in der reinikeit den lauteren Crystallen/werden zum mherentheil gefunden gegen mittag uff den höhsten und wilden bergen/die man nent schnee berg/(...)

Die jäger hencken das fleisch und wildprät darin zu summer zeyten/do mit es darin gefrier/(...)

Es braucht auch das landvolck den gletscher in tödlichen kranckheiten für artzney/(...)/unnd zu leschen acutas febres/(...).»

Trotzdem existiert aus der Mitte des 16. Jahrhunderts eine Beschreibung des Rhonegletschers, die wir der «Cosmographia» von MüNSTER (1628, p.691; s. auch lat. Ausgabe 1552, p. 332, 342) entnehmen können:

"Ich hab anno 1546 am 4. tag A ugusti einen (Gletscher, d. Verf.) gesehen bei der Fürcken/der ist bey zweyer oder dreyer Spiess dick/eines Armbrustschusses breit/der lenge mocht ich kein end uber sich seh:n/ist fürwar ein grawsames ansehen/es war ein stuck eines Hauss gross davon gefallen/das macht den Anblick noch grawsamerles gieng auch ein Bach mit wasser und Eyss darauss/dass ich mit meinem Ross ohn ein Brucken darüber nit kommen mochte. $\nu^{4}$

Aus dieser Beobachtung kann geschlossen werden, daß der Rhonegletscher sicher auf dem Talboden 
stirnte. Interessant ist die relativ genaue Beschreibung der Breite und der Höhe des Gletschers sowie die Erwähnung eines herabfallenden Eisstückes und des Baches - zweifellos ist damit die junge Rhone gemeint - mit eisführendem Wasser. Offensichtlich beschritt MÜNSTER den üblichen Furkaweg, der sich früher am linken Talhange entlang zog (vgl. Abb.1). Daß MÜNSTER ferner kein Ende des Gletschers ersehen konnte, ist der Form der Gletscherzunge zuzuschreiben, die dem Rhonegletscher bei größerer Ausdehnung eigen ist. Manchem Leser ist die eine oder andre Darstellung dieses Gletschers aus dem letzten Jahrhundert bekannt. Die etwas tatzenförmig aufgewölbte Gletscherzunge verunmöglichte, wäre man vor ihr gestanden, die Sicht gletscheraufwärts. Ebenso fällt dieser über eine Steilstufe ab und gestattet so auch keinen Blick in seine hintersten Eisregionen.

Wie groß nun genau die Ausdehnung des Rhonegletschers zu jener Zeit war, läßt sich aus diesem Hinweis nicht exakt eruieren. Nach LE ROY LADURIE (1967, p. 103ff.) muss eine größere Ausdehnung als nach 1930 angenommen werden. Ein wichtiger Anhaltspunkt, der eine Aussage in dieser Hinsicht zuläßt, kann aus neueren Untersuchungen am Großen Aletschgletscher (ноLZHAUSER in Vorber.) abgeleitet werden. Mit Hilfe von Feldbefunden und historischen Dokumenten (Alprechtsverträge) kann dort gezeigt werden, da $\beta$ der Gletscher schon ab den Jahren um 1520 die Ausdehnung von etwa 1920 aufwies. Einer Angabe von SCHINER (1812, p. 105) folgend, stießen die Saaser-Gletscher um diese Zeit merklich vor: «..., et surtout dans celle de Saas, où l'on prétend, que ces glaciers ont considérablement augmenté vers l'année 1529 (...)."

Vermutlich ist dieses Anwachsen der Gletscher auf eine klimatisch kühlere Phase, die mit einigen Unterbrüchen von etwa 1360-1470 dauerte, zurückzuführen (HOLZHAUSER in Vorber.).

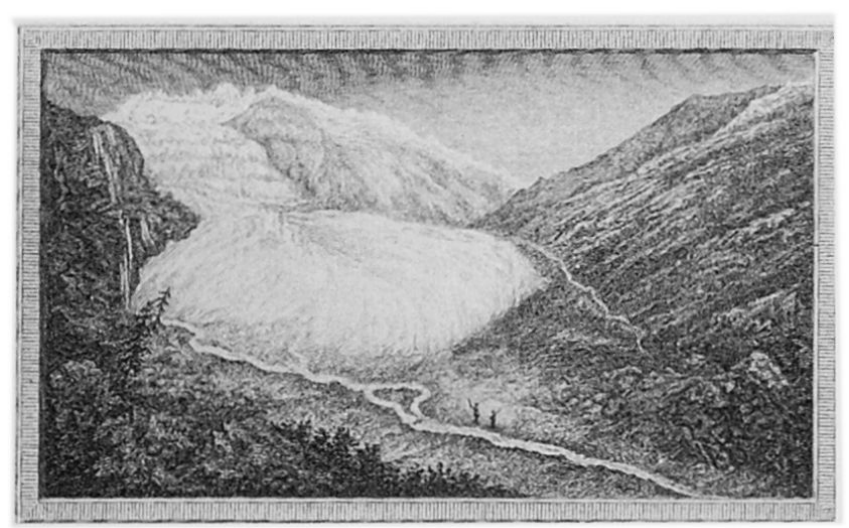

Abb. 1 Vue du Glacier du Rhône et de la Source de ce Fleuve. Radierung nach M.T. BOURRIT (vor 1781). In der rechten Bildhälfte ist der ehemalige Weg über die Furka erkennbar. (aus: BOURITT, m. t. 1781, Tome II, p. 6).
Einer näheren Erläuterung bedarf der Hinweis aus der Chronik von STUMPF (1554). Dort heißt es unter dem Jahre 1540 (8. Buch, 282): "Ein überheisser Summer/vom Mertzen bis Wyhenacht waerende/vertroechnet vil wasser und brunnen. Die schiffychen wasser wurdend wunder klein/so verbrunnend etliche waeld. Ein fruchtbar jar/gab vil wyns/und den über die massen guot in allen landen. Der Rhyn ward so klein, dass man under Basel zuo Rhynwyler dardurch reyten mocht. Zuo Mumpff under Seckingen fuor man mit den Landwaegen hinder den heüssern im Rheyn auff den trocknen sannd und nider. Die Lyndmat Zürych ward so klein/dass man das wasser aufschwellen/und mit gewalt an die Müli richten muosst auss der Schnelli.»

In einer etwas kürzeren Form findet man die Anmerkung über dieses besonders trockene Jahr in einem anderen Buch von STUMPF (1547/48): "Bei etlichen heissen Summers Zeyten als im Jahr Christi 1540 gewesen gadt auch etwan der alt Schnee ab, doch niemermeer also gar dann das die obristen Spitzen statigs Schnee behaltend."

Das Jahr 1540 muß demzufolge ein recht ungewöhnliches und aus der Reihe fallendes gewesen sein. Die Jahrzahl taucht in der Literatur später wieder auf, nämlich in einer Abhandlung über die Gletscher von HOTTINGER (1703, p. 49). Er erwähnt dieses trockene Jahr in seinen Ausführungen, wobei er sTuMPFs «Chronica» zitiert. Knapp fünfzig Jahre später berichtet altmann (1751, p. 23): "Die alten Gletscher bezeugen uns, dass alle Eisberge in der Schweiz im Jahre Christi 1540 mehr abgenommen als in soviel vorangehenden, weil man in dem Sommer derselben Jahrlaufs eine so grosse Hitze und Trockene verspühret, dass nicht nur alle Gletscher geschmolzen, sondern auch viele Eisberge von Schnee gänzlich entdecket und entblösset worden, so dass biss zur Herbstzeit der blosse Fels auf denselben zu sehen war, (...)."

Die Möglichkeit besteht, daß altmann sich auf HOTTINGER bezieht, möglich ist aber auch, daß er seine Angaben direkt der sTumpFschen Chronik entnommen hat und diese etwas ausgeschmückt wiedergibt. Denn die meiste Kenntnis von den Gebirgen basierte damals auf den Werken der Zürcher Gelehrten und neben dem, so in der Gelehrten Welt bekannte Herren Stumpf, Simmler, Gessner, Wagner, Hottinger und Scheuchzer uns gegeben, ist sehr weniges untersucht worden." (ALTMANN 1751, p.4/5). RICHTER (1891, p. 17) glaubt zwar, aus den Ausführungen altmanNs eine alte Chronik herauszuhören, die er aber nicht benennen könne. Vielleicht hatte ALTMANN Kenntnis einer alten Chronik. Für sein Werk über die «helvetischen Eisbergen» verwendete er, neben schon bestehenden Angaben, Hinweise, die er mittels Korrespondenz mit verschiedenen Personen sammelte, "die an unterschiedlichen Orten unsers Schweitzerlands nahe bey diesen Bergen wohnen". (ALTMANN 1751, p. 1). Aus eigener Anschauung kannte er die Grindelwaldgletscher, die er anläßlich einer Reise von nahe betrach- 
ten konnte und Hinweise vom damaligen Pfarrer über das Verhalten dieser Gletscher entgegennehmen konnte. Daraus erwuchs sein Wunsch, «eine Beschreibung der Eisbergen und Gletschern zu Papier zu bringen" (ALTMANN 1751, p.1). Möglich, daß er von einer Chronik wußte, die heute nicht mehr bekannt ist. Jedenfalls kann es sich nicht um die Grindelwaldchronik handeln, «welche für diese Zeit einen außergewöhnlich trockenen Sommer vermerkt, so daß ein Ausapern im Gebirge zweifellos eintreten konnte» (zUMBüHL 1980, p. 16). Aus der Fassung von altMaNN (s. auch GRUNER 1760), daß man «bis in den Herbst den nackten Fels gesehen habe», läßt sich vielleicht ein Eisfreiwerden des unteren Schopffelsbandes beim Unteren Grindelwaldgletscher herauslesen (zUMвÜнL 1980, p. 16). Daß aber alle Gletscher im Jahre 1540 abgeschmolzen waren, wie ALTMANN berichtet (siehe auch KASTHOFER 1822, p. 296/7), ist kaum anzunehmen und muß als Übertreibung gewertet werden.

\subsection{Das Vorstoßen der Gletscher gegen Ende des 16. Jahrhunderts}

Erste konkrete Hinweise über das Verhalten der Gletscher datieren aus der Zeit gegen Ende des 16. Jahrhunderts. Das damals einsetzende massive Vorstoßen der Gletscher schreckte manche Talbewohner auf. Die Eisströme nahmen bedrohliches Ausmaß an und begannen an einigen Orten, bebautes Land zu zerstören. So sollen sich die Verhältnisse nach HUG I (1842, zit. in zUмвёнL 1980, p. 17) beim Unteren Grindelwaldgletscher schon um 1565/72 geändert haben, was zur Folge hatte, daß dieser Gletscher um 1580 vorstieß. Aus verschiedenen Dokumenten ist eine große Gletscherausdehnung in den Jahren 1593-1606 belegt. Damals stieß der Untere Grindelwaldgletscher in Kulturland vor, was aus Bittschriften von 1603 hervorgeht (zUмвüHL 1980, p. 16ff.). Ebenso wiesen um 1580 die Gletscher im Vallée de Chamonix große Ausdehnung auf (BLEss 1979, p.8ff.). Erste Texte über Verwüstungen datieren von 1605 (LE ROY LADURIE 1967, p. 117).

Im Zusammenhang mit dem Vorrücken der Gletscher sind Nachrichten von Gletscherseeausbrüchen von Interesse. An vereinzelten Stellen dämmten von der Seite ins Haupttal mündende Gletscher mit zunehmendem Wachstum den Haupttalfluß ab, was zur Bildung eines Sees führte. Dieser konnte sich in unregelmäßigen Abständen entleeren und verheerende Überschwemmungen verursachen. Nachrichten von solchen Hochwasserkatastrophen, die nachweislich durch Ausbruch von Gletscherseen entstanden sind, lassen auf eine große Gletscherausdehnung schließen. Nach LüTsCHG (1926, p. 385) bewirkte ein Ausbruch des Mattmarksees, der vom Allalingletscher gestaut wurde, im Jahre 1589 eine Wasserflut im Saasertal (s. auch BIRCHER 1982). Ebenso schriftlich überliefert sind uns Ausbrüche des Lac du Ruitor im
Gebiet des oberen Aostatales aus der Zeit von 1594-1598 (BARETTI 1880, SACCO 1917, LE ROY LADURIE 1967, zit. in AESCHLIMANN 1979).

Ein weiteres Beispiel ist der Giétrogletscher im Val de Bagnes (sChNeEBeli 1976, RöthlisbeRger h. 1980) ${ }^{5}$. Dieser blockiert bei einem Hochstand durch einen am Fuß einer hohen Felswand sich bildenden Eiskegel die Dranse. Die so aufgestauten Wassermassen brachen von Zeit zu Zeit aus und drängten sich durch das Val de Bagnes hinunter bis ins Rhonetal nach Martigny, wo die Fluten zerstörerisch auf besiedeltes Gebiet einwirkten. Aus dem Jahre 1545 ist eine Hochwasserkatastrophe dieser Art überliefert (PERRIG CHRONIK, p. 680): "Im gedachten jahr (1545, d. Verf.) hat ein grosser Wasserguss in Bagne ob die 100 personen sambt viel gemächeren und Gütern jämmerlich untergemacht, daher diesem Thall ein Steur vom Land erfolget.» Nach SCHINER (1812, p.501), EBEL (1840, p.99) und FURRER (1852, Bd.2, p. 33) ereignete sich diese Katastrophe ebenfalls im Jahre 1545. Von einigen Autoren wird diese Jahrzahl jedoch angezweifelt. GILBERT (zit. in LüTsCHG 1926, p. 422) zum Beispiel meint, dass irrtümlicherweise 1545 anstatt 1595 (das Jahr einer späteren Überschwemmung, s. unten) geschrieben wurde. Auch courthion (1907, p. 79) hält diese Angabe für eine Verwechslung: «Mais aucun écrit positif ne vient attester cette catastrophe, que les dites chroniqueurs font remonter à 1545 . Si bien qu'on se demande si l'on confondrait pas cette date avec celle de 1595 , laquelle vit un grand débacle de la Dranse, analogue à celle de 1818.» Als unsicher muß auch ein angeblicher Ausbruch des Giétrosees am 7. August 1549 gewertet werden (sCHNEEBELI 1976, p. 23), denn er ist in keiner Chronik vermerkt. Es ist nicht auszuschließen, daß hier ein Übertragungsfehler (der Überschwemmung der Rhone vom 7.August 1469) vorliegen könnte (CHRONIQUE DE RIEDMATTEN, p. 16).

Gut dokumentiert ist hingegen der gewaltige Seeausbruch des Giétrosees im Jahre 1595. Eine Zusammenstellung der Hinweise kann bei LüTsCHG (1926) sowie bei COURTHION (1907) nachgelesen werden. In der CHRONIQUE DE RIEDMATTEN ist betreffs dieser Überschwemmung ein Eintrag vorhanden: "1595 25 May maximo inundatio aquarum ex Bagnes submergers martini» (p. 16).

Über den beginnenden Vorstoß der Gletscher am Ende des 16. Jahrhunderts berichtet auch ein Dokument aus Grächen (VS) und betrifft den Riedgletscher, der durch sein Vorrücken um 1600 eine Wasserleitung zerstörte (Gemeinde Grächen E 1, 1603; E 2, Abschrift von E 1 aus dem 17./18. Jahrhundert): «..., so hienach gemeldet werden, welche getheilen von alters her ein Wasser Furd, oder mer geschepfft uss dem Ryedbach und die selbigen gefiered uber ds Ryed gar und das Wasser geleittet uff ihren Berg Grechen Ihre gieter damit zu befychtigen, welche gelegenheit aber, und Wasserfad inen jetzt vor etlichen Jaren durch übertrang und nachtruck des wietenden Glötschers entzogen und 


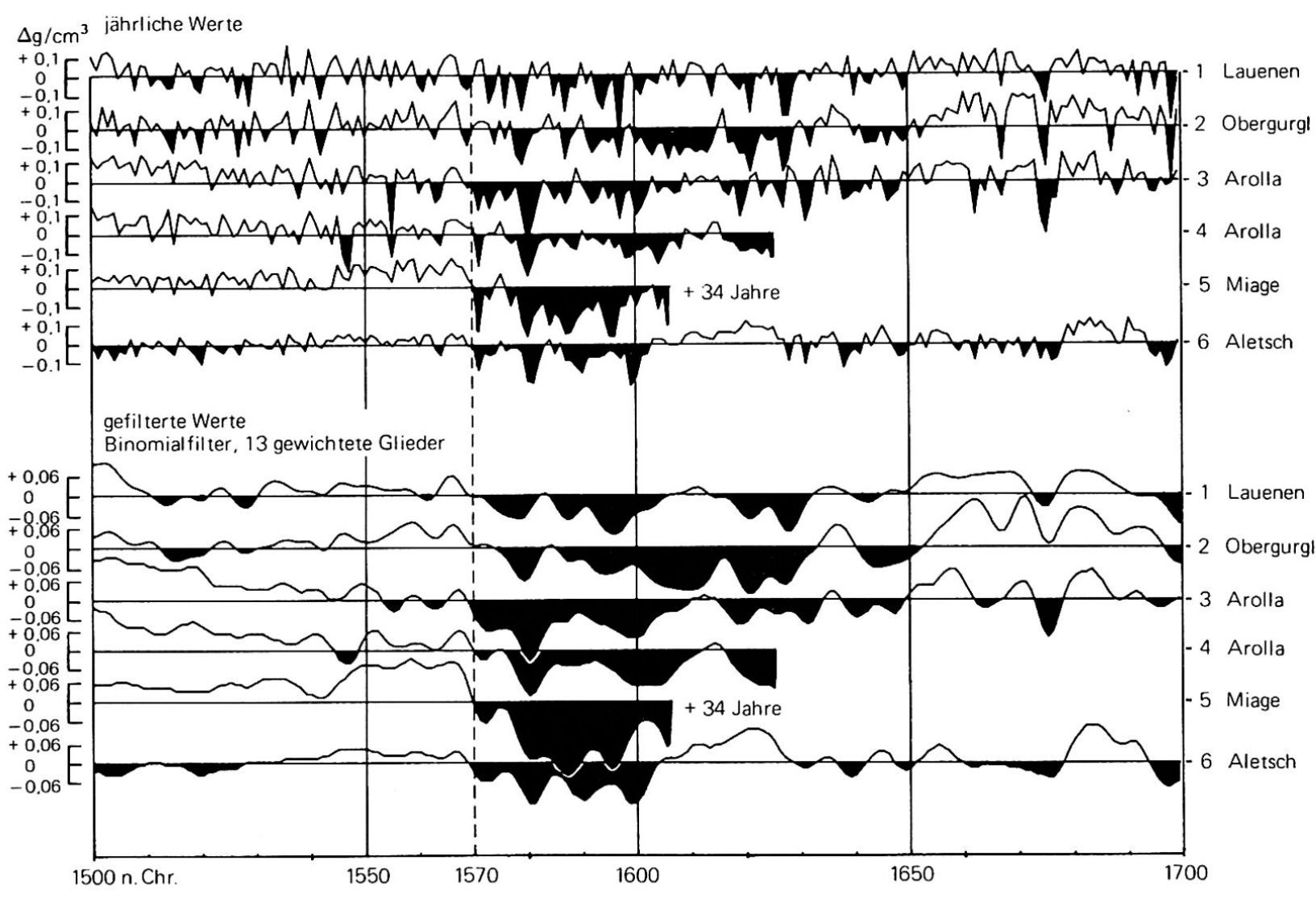

Kurve 1: Mittelkurve Lauenen (Berner Oberland), $1500 \mathrm{~m}$ ü. M. Absolute Chronologie (Fichte), Belegungsdichte: $10-25$ fach SCHWEINGRUBER et al. 1979

Kurve 2: Mittelkurve Obergurgl (Tirol), subalpine Lage. Absolute Chronologie (Fichte), Belegungsdichte: 10-20fach LAMPRECHT 1978

Kurve 3: EinzelkLirve (Lärche), Gletschervorfeld des Arollagletschers (VS) $2030 \mathrm{~m}$ ü. M.; Bergung und densitometrische Analyse: F.RÖTHLISBERGER

Kurve 4: Einzelkurve (Lärche), Gletschervorfeld des Arollagletschers (VS) $2030 \mathrm{~m}$ ü.M.; Bergung und densitometrische Analyse: F. RÖTHLISBERGER

Kurve 5: Einzelkurve (Lärche), Gletschervorfeld des Glacier de Miage (Südseite des Mont Blanc-Massivs) 1830 m ü. M.; Bergung des Holzes: H. AESCHLIMANN (Diss. in Vorb.)

Densitometrische Analyse: F. RENNER

Kurve 6: Einzelkurve (Lärche), Gletschervorfeld des Großen Aletschgletschers (VS) $1740 \mathrm{~m}$ ü. M.; Bergung des Holzes: H. HOLZHAUSER (Diss. in Vorber.)

Densitometrische Analyse: F. RENNER 
abgestossen ist, (...).» Um diese Gefahr abzuwenden und um weiteren Schaden zu verhindern, legten die Bewohner von Grächen ein Gelübde im Jahre $1639 \mathrm{ab}$ (schNidRig 1952, p. 30), das sieben Punkte beinhaltet. Dieses wurde 1803 neu verfaßt, vom Bischof gutgeheiBen und trägt den Titel "Regeln des Riedgletschers». (E 12/2, Grächen; Abschrift aus dem Jahre 1849 von J.P. Theler). Eine weitere und ähnlich lautende Abschrift entstand etwas später; das genaue Jahr ist nicht bekannt. Es hat z.B. den Zusatz, daß die Prozessionsteilnehmer «nüchtern bis zu dem Riedgletscher und wiederum heim gehen müssen" (E 12/3, Grächen). Nach Biffiger/walter (1973, p. 4) könnte diese Neufassung des Gelübdes mit einem erneuten Vorstoß des Riedgletschers in Zusammenhang gebracht werden, da der Gletscher 1862 die "Archa" (Wasserfassung) der "Chilcheri», die schon einmal im Jahre 1639 außer Betrieb war (E 7, St. Niklaus), bedrohte und das Wasser unter dem Gletscher gefaßt werden mußte, wobei ein Mann tödlich verunglückte. An dieser Stelle muß noch auf einen Fehler in der Arbeit von KINZL (1958, p. 225) hingewiesen werden. Im Kapitel «Die Gletscher zwischen 1000-1580» schreibt er von einem Vorstoß des Riedgletschers und dem damit verbundenen Gelübde im Jahre 1369. Richtigerweise sollte 1639 dafür stehen (vgl. scHNIDRIG, 1952, p. 30, ebenso E 12/3, Grächen).

Etwa um dieselbe Zeit zerstörte der Glacier de Durand (Zinalgletscher) im hinteren Val d'Anniviers den Weg, der zur Alp L'Ar Pitetta führte. In einem Dokument aus dem Jahre 1599 ist von der Wiederherstellung dieses Weges die Rede (LütschG 1926, RÖTHLISBERGER F. 1976, HAAS 1978).

Das soeben an einigen Beispielen dargelegte VorstoBen der Gletscher im gesamten Alpenraum gegen Ende des 16. Jahrhunderts ist die Folge einer Klimaverschlechterung. Nach PFISTER et al. (1978, p. 101) zeichnet sich eine ab 1560 einsetzende und über das Jahr 1600 hinausreichende grundlegende Veränderung der sommerlichen Witterungsverhältnisse ab, «die das Ausmaß landesweiter Ertragsschwankungen ausschlaggebend bestimmten" (PFISTER 1981, p. 491). Untersuchungen der Spätholzdichte an verbautem Holz im Berner Oberland («Lauenenkurve») ergaben, daß sich $a b 1570$ eine rapide Abkühlung einstellte und ein Wechsel von ähnlichen Sommern zu Sommern mit unterschiedlichem Charakter nachweisbar ist (schweIngruber et al. 1979). Daß dieser Abfall in der Spätholzdichte an Hölzern verschiedener Standorte feststellbar ist, soll Fig. 1 veranschaulichen (RENNER 1982, HOLZHAUSER in Vorber.).

\subsection{Die Gletscher im 17. Jahrhundert}

Die Frage, ob die Gletscher schon um oder kurz nach 1600 ihren ersten neuzeitlichen Hochstand erreicht haben, ist noch nicht ganz geklärt. Die zweitäußerste Moräne bei Gletsch (Rhonegletscher) wird gemeinhin als «1602er Moräne» bezeichnet. Diese Datierung geht auf MERCANTON (1916, p.49ff.) zurück, der diese Moräne in Analogie zu den Grindelwaldgletschern, die um 1600 vorstießen, zeitlich einstuft. ZUMBÜHL (1980, p. 18) schreibt, daß die gewaltigen Seitenmoränen beim Unteren Grindelwaldgletscher oberhalb des Marmorbruches und der Nellenbalm Material und eventuell Spuren dieses Vorstoßes um 1600 enthalten, daß aber eine eindeutige Abklärung des genauen Alters alleine mit Hilfe von historischen Dokumenten nicht möglich ist. Die Phase hoher Gletscherausdehnung dauerte lange an, da der Untere Grindelwaldgletscher 1669 noch (oder wieder) im Talboden lag (zUмвüHL 1980, p.81). Die äußersten Stirnmoränen entstanden bei diesem Gletscher und wahrscheinlich auch beim Oberen Grindelwaldgletscher in der Zeit dieser Maximalausdehnung (zимвӥнL 1980, p.82). $\mathrm{Ob}$ sich nun der Rhonegletscher gleich verhielt und die «1602er Moräne» auch einem Vorstoß um $1600 \mathrm{zu}$ zuordnen ist oder erst gegen Mitte des 17. Jahrhunderts zur Ablagerung gelangte, muß also vorerst noch mit einem Fragezeichen versehen werden.

Es scheint, daß sich auch bei anderen Gletschern in der ersten Hälfte des 17. Jahrhunderts eine erste neuzeitliche Maximalausdehnung über längere Zeit einstellte. So ist ein erster Hochstand der Gletscher im oberen Vallée de Chamonix (Glacier de Tour, Glacier d'Argentière) im Jahre 1610 überliefert, ein zweiter gegen das Jahr 1644. Im letztgenannten Jahr fand sogar eine Prozession zur Eindämmung der Gletschergefahr statt (BLEss 1979, p. 15/6). Diese Art der Gletscherbannung ist charakteristisch für diese Phase beginnenden Gletschervorstoßes und die daraus resultierende akute Gletschergefahr für Land und Leute. Die Prozession zum Mer de Glace in den Jahren 1643/44 hatte das gleiche Ziel, nämlich die um diesen Zeitpunkt anwachsende Gletschergefahr abzuwenden (wetTer 1979, p. 16). Aber nicht nur im Mont Blanc-Gebiet wurde auf religiöser Basis versucht, den hervordrängenden Gletschern Einhalt zu gebieten. In der Zeit um 1650 erreichten der Fiescher- und der Große Aletschgletscher maximale Ausdehnung. Im Falle des Fieschergletschers wurde ein Pater von Leuk damit beauftragt, den Gletscher durch heilige Zeremonien zu bannen: "Zuo 1652 war gefolget reverendus Michel Felliser von Leick ist wars 18 Monaten allhier gestorben. Dieser hat das gespenst im Viescher Gletscher beschworen, nach dem der Gletscher gestellt" (gestellt = stillgestanden, d. Verf./MICHEL CHRONIK, p. 19). Im September 1653 fand unter der Leitung von zwei Jesuiten-Patern ebenfalls eine Prozession zum GroBen Aletschgletscher statt. Aufgeschrieben ist diese Begebenheit im Buch «Historia Collegii Societatis Jesu Brigae in Vallesia» (handschriftliche Kopie im Kantonsarchiv Sitten, vgl. auch LÜTSCHG 1926, p. 387). Daraus geht hervor, dass der Große Aletschgletscher beträchtlich angewachsen war und die Weiden der Naterser bedrohte. Anscheinend erfüllte diese Gletscherbeschwörung ihren Zweck, denn der Gletscher stieß darauf nicht mehr weiter vor. 


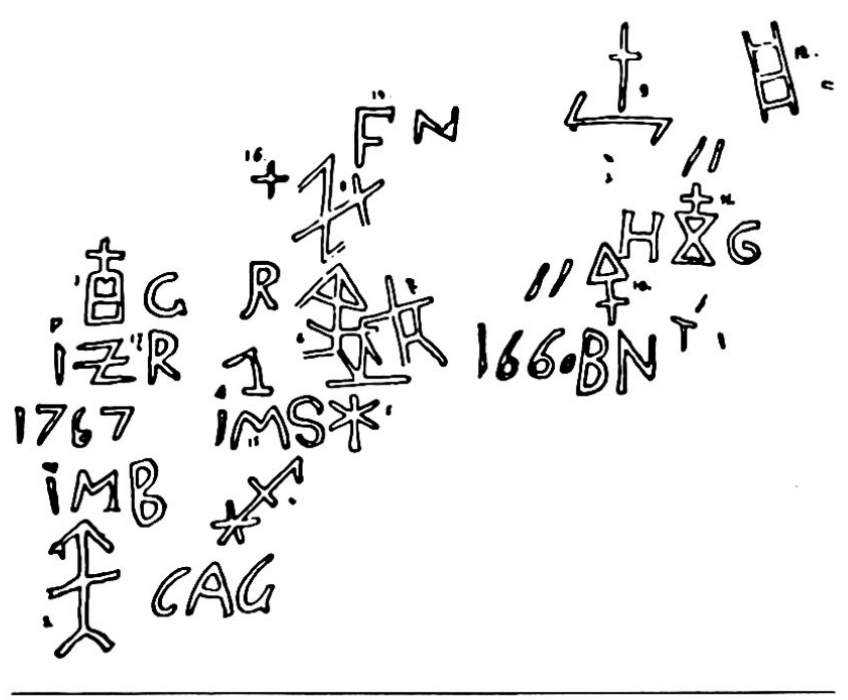

Abb. 2 Die Zeichen auf dem "Gletscherstein" auf der Göscheneralp mit den beiden Jahrzahlen 1767 und 1660. (aus: SCHALLER-DONAUER, a. 1925).

Als Hinweis für einen Gletschervorsto $ß$ um diese Zeit darf vielleicht auch der Gletscherstein oder «hübsche Stein» auf der Göschener Alp gelten (sCHALLER DONAUER, 1925, p. 300-301). Dieser Stein, gelegen am Fuß einer Moräne am Eingang ins Wintergletschertal, trägt eine Menge von Hauszeichen und Initialen. Darunter befinden sich auch noch die zwei Jahreszahlen 1660 und 1767. Diese beziehen sich mit großer Wahrscheinlichkeit auf Jahre, in denen eine Wallfahrt durchgeführt wurde, um den vorrückenden Gletscher (Dammagletscher) aufzuhalten und um die Weiden zu schützen. Die Initialen und Hauszeichen stammen größtenteils von Leuten der Göschener Alp, die an den Wallfahrten teilnahmen (Abb. 2).

Nach diesem ersten neuzeitlichen Hochstand schmolzen die Gletscher allgemein wieder etwas ab, doch nicht um allzu große Beträge. Um 1669 ist beim Unteren Grindelwaldgletscher ein kurzer Vorstoß anzunehmen; dieser ist aber weder zeitlich noch topographisch genau abgrenzbar (zимвüHL 1978, p. $99 ; 1980$, p. 20). Beim Pré de Bar-Gletscher (Mont Blanc-Gebiet) sind Vorstöße um 1676 und um 1688 belegt (AESCHLIMANN 1979, p. 59).

\subsection{Die Gletscher im 18. Jahrhundert}

Das 18. Jahrhundert begann mit einer Phase, in der sich die Gletscher wiederum ausdehnten und hochstandsähnliche Ausmaße erreichten. Die Grindelwaldgletscher zum Beispiel begannen mit dem Vorrücken um 1705 und erreichten um 1720 den Kulminationspunkt. Um 1750 jedoch ist bei diesen Gletschern durch Schrift- und Bildquellen ein Ausapern belegt, die Gletscherzungen waren stark zurückgeschmolzen (zимвühl 1980, p.25). Auch beim Großen Aletsch- gletscher ist eine geringere Ausdehnung als um 1650 in den Jahren um 1755 festzustellen (HOLzHAUSER 1980, p. 17ff.), ebenso im Jahre 1749 und 1793 (HolzHAUSER/WETTER 1982).

In dieser Richtung darf vielleicht auch eine Angabe von venetz (1821, p. 10) gedeutet werden, daß nämlich der Rothwelschgletscher (heute Hübschhorngletscher) oberhalb des Simplonpasses im Jahre 1732 nur in Form einer kleinen Anhäufung von Schnee bestanden haben soll. (In der "Statistik und Urkundensammlung über Wallis» von FURRER [1852 Bd.2, p. 47] steht vermutlich irrtümlicherweise die Jahrzahl 1432.) Ebenso hatte der Gornergletscher bei Zermatt eine kleinere Ausdehnung in der zweiten Hälfte des 18. Jahrhunderts (GUNTERN 1979, p.46/Nr. 44): An einer Stelle, wo in jener Zeit ein Landgut mit einem Haus stand, liegen jetzt die Moränen der Vorstöße des 19. Jahrhunderts. TYNDALL (1898, p. 147), der den Gornergletscher am 8. August 1858 besuchte, schreibt, daß ihm ein Wirt bei dieser Gelegenheit erzählte, daß dieser Gletscher in den letzten sechzig Jahren vierundvierzig Sennhütten umgestürzt hat. Kleinere Ausdehnungen der Gletscher im unteren Vallée de Chamonix um 1760 lassen sich auch aus Beschreibungen von SAUSSURE $(1779,1786)$ ableiten (wetTER 1979, p. 19, 20, 39). Daß im 18. Jahrhundert längere Phasen günstigere klimatische Bedingungen geherrscht haben müssen, ist einer Arbeit von PFISTER (1981, p. 484ff.) zu entnehmen, wonach in den Jahren 1718-1729 fünf Spitzenernten zu verzeichnen waren, was zum Begriff der «goldenen Zwanzigern» (p. 485) führte. Auch die Zeitspanne 1740-1773, die durch keine besonderen Merkmale gekennzeichnet war, wies eine Phase überdurchschnittlicher Produktion auf. Das Vierteljahrhundert von 1774-1798 stand unter dem Zeichen eines lang anhaltenden «Weinbooms». Diese günstigeren Jahre verhinderten jedoch ein erneutes Anwachsen der Gletscher gegen 1780 nicht. Gut dokumentiert ist dieser Vorsto $B$ bei den Grindelwaldgletschern (zимвӥнц 1978, p.99; 1980, p. 26ff., p.61ff.) und bei den Gletschern im Mont Blanc-Gebiet (aEsChLIMANN 1979, Bless 1979, wetter 1979). Die Schwankungen der Gletscher in dieser Zeit können nach Untersuchungen von PFISTER $(1980$, p. 188) kurzfristigen Schwankungen der Sommertemperaturen zugeordnet werden.

\subsection{Die Gletscherhochstände im 19. Jahrhundert und der Rückzug nach 1850}

Zu den kältesten und nässesten Perioden seit dem 17. Jahrhundert gehört die Zeitspanne von 1812 bis 1817 (PFISTER 1980, p. 188; 1981, p. 487). Auch die ChroniK VON EISTEN weiß von Hungersnöten in den Jahren 1816 und $1817 \mathrm{zu}$ berichten. Nässe und Kälte sollen im Jahr 1817 so andauernd gewesen sein, daß die Bäume oberhalb $1800 \mathrm{~m}$ ü. M. gar nicht mehr zum Grünen kamen. Begleitet waren diese Jahre von 
massiven Gletschervorstößen, bei denen die Gletscher zum größten Teil bis nahe an die Moränen des 17. Jahrhunderts heran reichten. Bei den Grindelwaldgletschern wurde mehr Talboden von Eis bedeckt als beim letzten Vorstoß im 18. Jahrhundert (ZUмBüHL 1978, p. 99).

In diese Zeit, nämlich ins Jahr 1818, fällt auch ein Ausbruch des Giétrosees (sCHNeEbeli 1976, p. 23; RÖTHLISBERGER H. 1978, p. 200ff.).

Die Hochstandsphase der Gletscher dauerte die ganze erste Hälfte des 19. Jahrhunderts. Nach einem kurzen Rückschmelzen, das dem Hochstand um 1820 folgte, wurde eine zweite Kulmination um die Jahre 1850 erreicht. Das Ausmaß dieser beiden Vorstöße variiert unter den einzelnen Gletschern. Zum Beispiel war der Vorstoß beim Glacier de Brenay und beim Glacier du Mont Durand (beide Wallis) um 1820 größer (sCHNEEBELI 1976, p.56), ebenso beim Oberen Grindelwaldgletscher (zимвӥнц 1980, p. 72), beim Mer de Glace und beim Glacier des Bossons im unteren Vallée de Chamonix (wetter 1979). Hingegen erreichten der Oberaargletscher (AMMANN 1978, p. 281), der Untere Grindelwaldgletscher (zчмвӥнL 1980, p. 52), der Aletsch- und der Fieschergletscher (hOLzHAuser 1978 , p. 51ff.; 1980 , p. 22) ihr zweites neuzeitliches Maximum um 1850.

Nach 1850 setzte eine langfristige Abschmelzphase ein, die durch einen beträchtlichen Gletscherschwund gekennzeichnet ist, der beim größten Teil der Gletscher nur durch kleinere Vorstöße (hauptsächlich um $1880 / 90$ und um 1920) kurz unterbrochen wurde.

Durch genauere Beobachtungen, vor allem durch die Gletschervermessung ab 1880 , sind wir über das Gletscherverhalten in diesem Zeitabschnitt gut unterrichtet (Zusammenstellung S. AELLEN/KASSER 1981).

\subsection{Zusammenfassung}

Betrachten wir zusammenfassend die Gletschergeschichte der Neuzeit, so ergibt sich folgendes Bild (vgl. Fig. 2):

Die gegen Ende des 16. Jahrhunderts einsetzende Klimaverschlechterung, die sich über mehrere Jahrzehnte hinweg erstreckte («long term climatic fluctuation", S. PFISTER et al. 1978, p. 100), verursachte ein gewaltiges Vorstoßen der Gletscher. Einige erreichten in der ersten Hälfte des 17. Jahrhunderts ihren neuzeitlichen Maximalstand. Während der nun folgenden 250 Jahre bewegten sich die Gletscher nicht mehr sehr weit zurück. Sie oszillierten im Bereich der Maximalmoränen, so daß allgemein ausgedrückt für die Zeit von 1600-1850 von einer Hochstandsphase der Gletscher gesprochen werden kann.

Das 18. Jahrhundert ist gekennzeichnet durch Phasen geringerer Ausdehnung der Gletscher als im 17. und im darauffolgenden 19. Jahrhundert mit Vorstößen um 1720 und um 1780. Das 19. Jahrhundert beginnt mit einer kurzen, aber intensiven Klimaverschlechterung, die Gletscherhochstände um 1820 und um 1850 bewirkte. Seit diesem letzten Hochstand zeichnet sich ein langfristiges Abschmelzen der Gletscher ab, das nur durch kurze Rückzugshalte oder Vorstöße unterbrochen wurde.

\section{Schlußbemerkung}

Untersuchungen von Eisbohrkernen in Grönland zufolge soll sich, nach einem mittelalterlichen Klimaoptimum (ca.950-1200), schon um 1250 A.D. eine Klimawende eingestellt haben (schöNwIESE 1979, p. $75 \mathrm{ff}$.), die dann zum sogenannten «Little Ice Age» überleitete. Beim Großen Aletschgletscher ist in der Zeit um 1300 ein Vorstoß mit anschließendem Hochstand belegt. (FURRER et al. 1982, HOLZHAUSER in Vorber.) LAM B (1977) bezeichnet die Übergangsphase zum "Little Ice Age» als Klimawende des späten Mittelalters und setzt den Beginn der «Kleinen Eiszeit) um 1430 fest (gribBin/LAmB 1978, p. 70). Nach FLOHN (1978) dauert diese kühle Phase von 1550-1850, wobei er sich für die zeitliche Abgrenzung am Verhalten der Alpengletscher orientiert, was durchaus sinnvoll erscheint. Nach PFISTER (1980, p. 187) setzt die Klimaverschlechterung um 1560 ein, was auch mit dendrochronologischen Befunden gut übereinstimmt (s. Kap. 2.2).

Ob der Begriff "Little Ice Age» für die Benennung der rund 350 Jahre dauernden Hochstandsphase der Gletscher angemessen ist, darüber sollte man sich vielleicht doch einige Gedanken machen. Diese Bezeichnung geht auf matthes $(1939,1940,1949)$ zurück und umfaßte ursprünglich einen Zeitraum von ca. 4000 Jahren. Diese Annahme stützte sich auf Untersuchungen von ANTEVs (1938) und steht auch im Zusammenhang mit der alten Auffassung einer postglazialen Wärmezeit (vgl. Zusammenstellung bei PATZelt 1980, p. 12). MATthes (1949, p. 214): «..., and accordingly it may well be said that we are living in an epoch of renewed but moderate glaciation - a «little ice age», that already lasted about 4000 years.»

In der Folgezeit wurde dieser Begriff für einen viel kürzeren Zeitabschnitt übernommen, nämlich nur noch für die rund 350 Jahre dauernde Hochstandsphase der Gletscher während der Neuzeit. Es stellt sich die Frage, ob diese Benennung angemessen ist, wissen wir doch, daß während der Dauer des gesamten Postglazials die Gletscher mehrmals die Ausmaße von 1850 erreichten. Konsequenterweise müßte jede dieser Vorstoßphasen mit "Little Ice Age» umschrieben werden. Schon LE ROY LADURIE (1967, p. 211) schrieb von einer "distorsion» dieses Begriffes und schlug vor, diese letzte große Vorstoßphase «phase multiséculaire ultime de crue des glaciers» oder, in Anlehnung an KINZL (vgl. Kap. 1), kurz "Fernau» zu nennen. Auch Patzelt (1980, p. 16) bemerkt: «Die Bezeichnung einer "Kleinen Eiszeit" für diese Periode, wie sie in der englischen Literatur üblich ist 


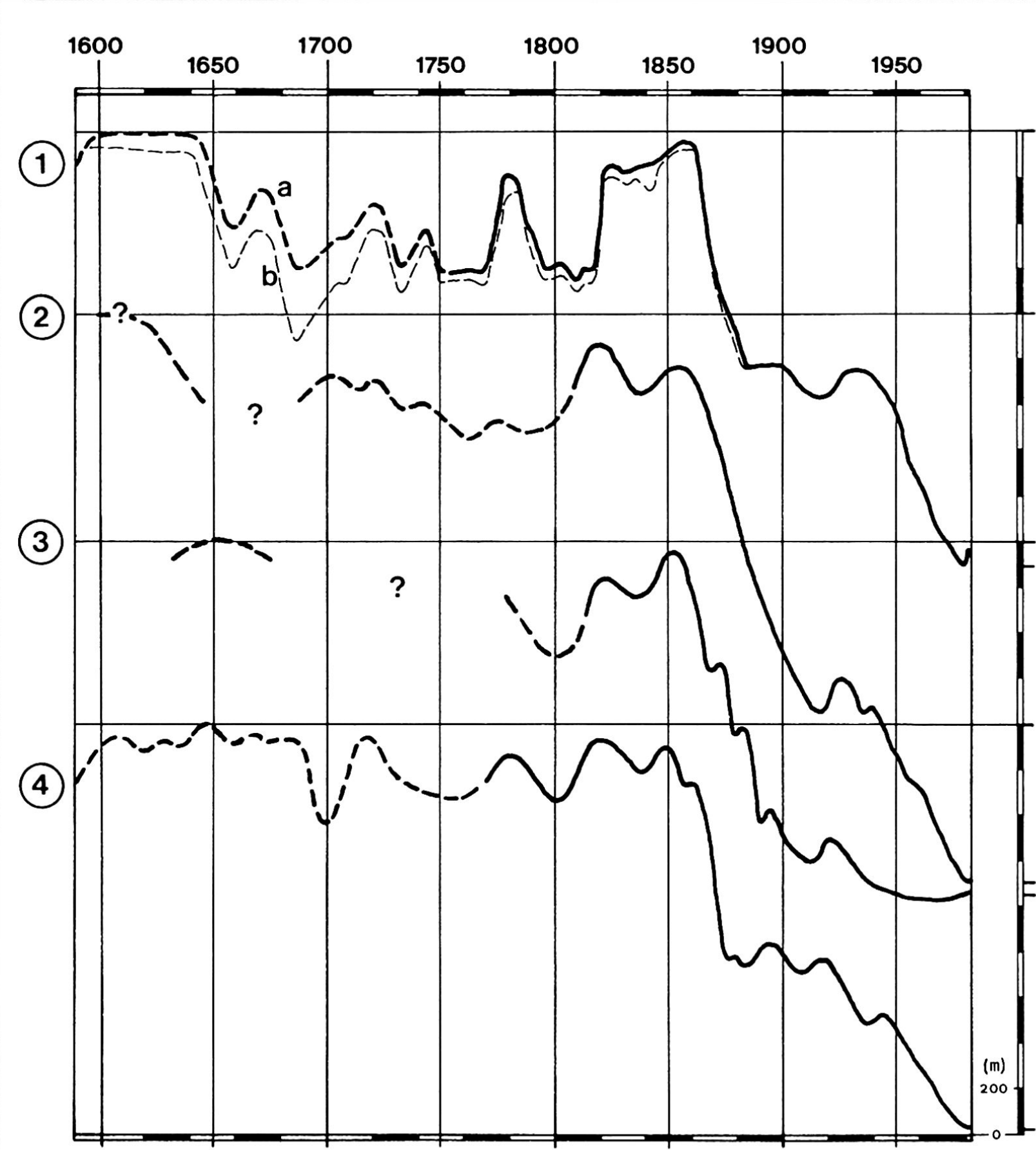

1 Unterer Grindelwaldgletscher (a: Maximal-, b: Minimalwerte) nach ZUMBÜHL (1978, 1980), vereinfacht.

2 Rhonegletscher, nach MERCANTON (1916)

3 Fieschergletscher, nach HOLZHAUSER (1978)

4 Mer de Glace (Chamonix), nach WETTER (1979) 
und vielfach übernommen wurde, ist in keiner Weise gerechtfertigt.»

Vielleicht wäre es besser, die Zeitspanne vom beginnenden Vorrücken der Gletscher gegen Ende des 16. Jahrhunderts bis zum Abklingen des letzten Hochstandes um 1850 als "Gletscherhochstandsphase der Neuzeit" zu benennen.

Eine Frage, die noch unbefriedigend geklärt ist und in dieses Kapitel Gletschergeschichte gehört, ist diejenige nach der Ausgangslage der Gletscher vor der Klimaverschlechterung um 1560/70. PFISTER et al. (1978, p. 104) postulieren, «daß die Gletscher in jenem Zeitpunkt hinter die Ausgangspositionen zurückgeschmolzen waren, die sie in den folgenden drei Jahrhunderten jeweils zwischen zwei Vorstößen einnahmen».

Aufgrund der Hinweise in Kapitel 2.1 darf angenommen werden, daß die Gletscher mit großer Wahrscheinlichkeit ausgedehnter waren als heute, und dies vermutlich, wie neuere Untersuchungen zeigen (HOLzhauser in Vorber.), seit Beginn des 14. Jahrhunderts.

\section{Anmerkungen}

${ }^{1}$ Unter Gletscherausdehnung verstehen wir einen momentanten Umriß des Gletschers. Gletscherstand bezeichnet einen Gletschervorstoß oder einen längeren Gletscherhalt mit Moränenbildung. Ein Hochstand (im Verlauf des Postglazials) bedeutet einen Gletschervorstoß in der Größenordnung von 1850. Die Bezeichnung Maximalstand bezieht sich auf den ausgedehntesten Hochstand in der jeweiligen Hochstandsphase.

${ }^{2}$ Bei den im Text genannten Jahreszahlen handelt es sich um Jahre n. Chr.

${ }^{3}$ Erste gedruckte Schweizerkarte von M. WALDSEEMÜLLER (gezeichnet 1508, gedruckt 1513); Karten von G. TSCHUDI, J.STUMPF Und S. MÜNSTER, alle 1538.

${ }^{4}$ Die Reise von S.MÜNSTER ist auch in der PERRIG CHRONIK (hier Sebastianus Monsterus genannt) vermerkt unter der Jahrzahl 1547 (p. 684).

${ }^{5}$ In diesem Zusammenhang soll ein Hinweis aus der Vorneuzeit kurz erwähnt werden. Um 580 wurde der Bischofssitz von Martigny (auf La Batiaz) nach Sion (Sitten) verlegt. Veranlassung dazu soll ein Ausbruch des Giétrosees gegeben haben (schNeEBELI 1976, p. 23). Wie aber bei GREMAuD (1878, Bd.I, p. 11 , $\mathrm{Nr}$. 15) nachzulesen ist, trat in besagtem Jahre die Rhone über die Ufer (und nicht die Dranse): "Anno I Cons. Tiberii Constantini Aug. Ind.XIII. Eo anno, mense octobre, ita in Vallensi territorio Rhodanus exundavit, ut copias messium denegaret." Was die Verlegung des Bischofsitzes betrifft, so spielten politische Überlegungen eine Rolle. In der PERRIG CHRONIK steht zum Jahre 579 folgende Notiz (p. 327/28):

"In diesen unruhigen Zeiten nun der Langobarden hatte sich unser Bischoff Agricola Landhinauf in die Sicherheit geflüchtet wegen dessen nun das man etlich nachher erfahren, dass Martinach an einem solchen pass und angang wo gleich der Bischoffliche Sitz und Würde in Gefahr stünde, ist gedachter Sitz zweifel ohne durch unseren König Gontramann auf Sitten als einen sicheren und noch mit gut eingeschlossenen Mauren eingeschlossenen Ort, (dan zu Martinach alles niedergerissen war) versetzt und transferiert worden und ist der erste dort zu Sitten Bischoff erwählt worden, und sich Bischoff von Sitten beschrieben und geheissen.»

Nach CARLEN (1981, p. 11) waren neben praktischen seelsorgerischen $G$ ründen ebenfalls politische Gründe (Langobardeneinfall über den Großen St. Bernhard im Jahre 574) für die Verlegung des Bischofsitzes maßgebend.

Die Annahme eines Hochstandes des Giétrogletschers in jener Zeit ist somit nicht berechtigt.

\section{Literaturverzeichnis}

\section{Gedruckte Quellen und Sekundärliteratur}

AELLEN, M./KASSER, R. (1981): Les variations des glaciers suisses en 1979/80. Rapport préliminaire. Section de glaciologie de la Société hydrotechnique de France, Réunion des 12 et 13 mars 1981 à Grenoble.

AESCHLIMANN, H. (1979): Neuzeitliche Gletschergeschichte im Mont Blanc-Massiv. Val Veni-Val Ferret-Ruitor. Diplomarbeit, Manuskr. Geogr. Inst. Univ. Zürich.

ALTMANN, J.G. (1751): Versuch einer historischen und physischen Beschreibung der helvetischen Eisbergen. Zürich.

AMMANN, K. (1978): Der Oberaargletscher im 18., 19. und 20. Jahrhundert. In: Zeitschr. für Gletscherkunde und Glazialgeologie, Bd. XII, H. 2: 253-291. Innsbruck.

ANTEVS, E. (1938): Post-pluvial climatic variations in the Southwest. In: Bull. Amer. Met. Soc., Vol. 19: 190-193.

BARETTI, M. (1880): Il Lago di Rutor. Alpi Graje Settentrionali. In: Boll. del C. A. I., Nr. 51. Torino.

BIFFIGER, K.WALTER, R. (1973): Die Regeln des Riedgletschers. In: Wir Walser. Halbjschr. für das Walsertum, 11.Jg., Nr. 1, 1-9. Visp.

BIRCHER, W. (1982): Beiträge zur Gletscher- und Klimageschichte des Saastales. Glazialmorphologische und dendroklimatologische Untersuchungen. Dissertation Univ. Zürich.

BLESS, R. (1979): Neuzeitliche Gletschergeschichte im Mont Blanc-Massiv. Valée du Trient-oberes Vallée de Chamonix. Diplomarbeit, Manuskr. Geogr. Inst. Univ. Zürich.

BOURRIT, M.T. (1781): Description des Alpes Pennines et Rhétiens, Tome II. Genève.

BÜHLER, A. (1928): Das Meiental im Kanton Uri. Bern.

CHARLESWORTH, J.K. (1957): The Quaternary Era, with special reference to its glaciation. London.

COURTHION, L. (1907): Bagnes-Entremont-Ferrex. Guide pittoresque et historique. Genève. 
EBEL, J.G. (1840): Anleitung, auf die nützlichste und genußvollste Art die Schweiz zu bereisen. Bearbeitet von G.von ESCHER. Zürich.

FINSTERWALDER, S. (1897): der Vernagtferner. Wissenschaftliche Ergänzungshefte zur Zeitschr. des Deutschen und Österreichischen Alpenvereins, 1. Heft.

FLOHN, H. (1978): |Die Zukunft unseres Klimas: Fakten und Probleme. Promet 2/3: 1-21.

FURRER, G./GAMPER-SCHOLLENBERGER, B./SUTER, J. (1978): Zur Geschichte unserer Gletscher in der Nacheiszeit. Methoden und Ergebnisse. In: Jahrbuch der Schweiz. Naturforsch. Ges., wissenschaftlicher Teil: 76-88. Birkhäuser Verlag, Basel/Boston/Stuttgart.

FURRER, G./HOLZHAUSER, H./MAISCH, M./SUTER, J. (1982): Zur Geschichte unserer Gletscher im Spät- und im Postglazial. In: Unversität Zürich, Berichte aus der Forschung. Zum 150-Jahr-Jubiläum der Univ. Zürich, Hrsg. vom Wissenschaftl. Informationsdienst: 103-114.

FURRER, S. (1850/1852): Geschichte, Statistik und Urkundensammlung über Wallis, 3 Bde., Sitten.

GAMPER, M./SUTER, J. (1982): Postglaziale Klimageschichte der Schweizer Alpen. Dieses Heft.

GILBERT, L.W. (1819): Annalen der Physik. Jg. 1818, Bd.60, 12. Stück. Leipzig.

GREMAUD, J. (1878): Documents relatifs à I'histoire du Valais. Tome I. Lausanne.

GRIBBIN, J./LAMB, H.H. (1978): Climatic change in historical times. In: Climatic change: 68-82. Cambridge University Press.

GRUNER, G. S. (1760): Die Eisgebirge des Schweitzerlandes. 3 Bde. Bern.

GUNTERN, J. (1979): Volkserzählungen aus dem Wallis. 2. Auflage. Basel.

HAAS, Ph. (1978): Untersuchungen zur Gletschergeschichte im Val d'Anniviers. Diplomarbeit, Manuskr. Geogr. Inst. Univ. Zürich.

HOLZHAUSER, H. (1978): Zur Geschichte des Fieschergletschers. Diplomarbeit, Manuskr. Geogr. Inst. Univ. Zürich.

HOLZHAUSER, H. (1980): Beitrag zur Geschichte des Großen Aletschgletschers. In: Geographica Helvetica, 35.Jg., Nr.1: 17-24. Kümmerly \& Frey. Bern.

HOLZHAUSER, H.METTER, W. (1982): Auswertung historischer Quellen zur jüngsten Gletschergeschichte. In: M. GAMPER: Beiträge zur Quartärforschung in der Schweiz. Physische Geographie, Vol. 1. Zürich.

HOLZHAUSER, $\mathrm{H}$. in Vorber.: Zur Geschichte des Fiescherund der Aletschgletscher.

HOTTINGER, J.H. (1703): Montium glacialium helveticorum descriptio.

HUGI, F.J. (1842): Über das Wesen der Gletscher und Winterreise in das Eismeer. Stuttgart/Tübingen.

IMHOF, E. (1974): Kartographische Rekonstruktion früherer landschaftlicher Zustände. In: Festschrift für Georg Jensch, Abhandl. des 1.Geogr. Inst. der Freien Universität Berlin, Bd. 20: 129-138.

KASTHOFER, K. (1822): III. Betrachtungen über die Veränderungen in dem Klima des Alpengebirgs: 221-270. Aarau.

KINZL, H. (1929): Beiträge zur Geschichte der Gletscherschwankungen in den Ostalpen. In: Zeitschr. für Gletscherkunde, Bd. XVII, H. 1-3: 66-121. Leipzig.

KINZL, H. (1932): Die größten nacheiszeitlichen Gletschervorstöße in den Schweizer Alpen und in der Mont Blanc-Gruppe.
In: Zeitschr. für Gletscherkunde, Bd.XIX, H. 4-5: 269-397 (Finsterwalder Festschrift). Leipzig.

KINZL, H. (1949): Formenkundliche Beobachtungen im Vorfeld der Alpengletscher. In: Sonderdruck aus Veröff. des Museum Ferdinandeum (Innsbruck), Bd.26/29, Jge. 1946/49: 61-82 (Klebelsberg Festschrift). Innsbruck.

KINZL, H. (1958): Die Gletscher als Kimazeugen. In: Deutscher Geographentag 1957: 222-231.

LAMB, H.H. (1977): Climate: present, past and future, Vol. 2. Methuen, London.

LAMPRECHT, A. (1978): Die Beziehungen zwischen Holzdichtewerten von Fichten aus subalpinen Lagen des Tirols und Witterungsdaten aus Chroniken im Zeitraum von 1370-1800 A. D. Diplomarbeit, Manuskr. Geogr. Inst. Univ. Zürich.

LE ROY LADURIE, E. (1967): Histoire du climat depuis l'an mil. Flammarion, Paris.

LUCERNA, R. (1913): Die Flächengliederung der Mt.BlancGruppe. Geogr. Zeitschr.

LÜTSCHG, O. (1926): Über Niederschlag und Abfluß im Hochgebirge. Sonderdarstellung des Mattmarkgebietes. Ein Beitrag zur Fluß- und Gletscherkunde der Schweiz. Schweizer Wasserwirtschaftsverband. Verbschr. N 14. Zürich.

MAISCH, M. (1981): Glazialmorphologische und gletschergeschichtliche Untersuchungen im Gebiet zwischen Landwasserund Albulatal (Kt. Graubünden, Schweiz). Dissertation Univ. Zürich.

MAISCH, M. (1982): Zur Gletscher- und Klimageschichte des Spätglazials. Dieses Heft.

MATTHES, F.E. (1939): Report of Committee on Glaciers, April 1939. In: National Research Council, American Geophysical Union Transactions of 1939: 518-523. Washington.

MATTHES, F.E. (1940): Report of Committee on Glaciers, 1939-40. In: National Research Council, American Geophysical Union Transactions of 1940: 396-406 Washington.

MATTHES, F.E. (1949): Glaciers. In: Hydrology, edited by O. MEINZER. New York.

MERCANTON, P.L. (1916): Vermessungen am Rhonegletscher, 1874-1915. In: Neue Denkschr. der Schweiz. Naturforsch. Ges., Bd. 52. Basel/Gent/Lyon.

MOUGIN, M. (1912): Etudes glacialogiques en Savoie. Etudes glaciologiques Tome III. Ministère de l'agriculture.

MÜNSTER, S. (1544): Cosmographia; Beschreibung aller Länder durch Sebastianum Munsterum, ..., Basel.

MÜNSTER, S. (1550): Cosmographia universalis, Basilea.

MÜNSTER, S. (1552): Cosmographia universalis, Lib. VI, Basilea.

MÜNSTER, S. (1628): Cosmographia, das ist Beschreibung der ganzen Welt, darinnen aller Monarchien, Keyserthumben, Königreichen, ..., Ursprung, Regiment, ... durch ... Sebastianum Munsterum... Auff das newe ubersehen und mit vielerley nothwendigen Sachen .. vermehrt. 4 Bde., Basel.

NANGERONI, G. (1927): Osservazioni su alcuni ghiacciai della Valtournanche (Grandes Murailles). Boll. Com. Glac., 7.

PATZELT, G. (1980): Neue Ergebnisse der Spät- und Postglazialforschung in Tirol. In: Jahresbericht der Österr. Geogr. Gesellschaft, Zweig Innsbruck, 1976/77: 11-18.

PFISTER, Ch./MESSERLI, B./MESSERLI, P./ZUMBÜHL, H. (1978): Die Rekonstruktion des Klima- und Witterungsverlaufes der letzten Jahrhunderte mit Hilfe verschiedener Datentypen. In: Jb. der Schweiz. Naturforsch. Ges., wissenschaftlicher Teil: 89-105. Birkhäuser Verlag, Basel/Boston/Stuttgart. 
PFISTER, Ch. (1980): Klimaschwankungen und Witterungsverhältnisse im Mittelland und Alpenvorland zur Zeit des "Little Ice Age». Die Aussage der historischen Quellen. In: Das Klima: 175-190. Springer-Verlag, Berlin/Heidelberg/New York.

PFISTER, Ch. (1981): Die Fluktuationen der Weinmosterträge im schweizerischen Weinland vom 16. bis ins frühe 19. Jahrhundert. Klimatische Ursachen und sozioökonomische Bedeutung. In: Schweiz. Zeitschr. für Geschichte, Bd. 31: 445-491. Basel.

RENNER, F. (1982): Beiträge zur Gletschergeschichte des Gotthardgebietes und dendroklimatologische Untersuchungen an fossilen Hölzern. Dissertation Univ. Zürich.

RICHTER, E. (1891): Geschichte der Schwankungen der Alpengletscher. In: Zeitschrift des Deutschen und Österreichischen Alpenvereins, Bd. 22: 1-74.

RÖTHLISBERGER, F. (1976): Gletscher- und Klimaschwankungen im Raum Zermatt, Ferpècle und Arolla. In: Die Alpen, 52.Jg., Nr. 3/4: 59-152. Bern.

RÖTHLISBERGER, H. (1978): Eislawinen und Ausbrüche von Gletscherseen. In: Jahrbuch der Schweiz. Naturforsch. Ges., wissenschaftlicher Teil: 170-212. Birkhäuser Verlag, Basel/Boston/Stuttgart.

SACCO, F. (1917): L'Apparato Morenico del Ghiacciaio del Miage. In: Boll. della soc. geol. italiana, Bd.XXVI: 323-354. Roma.

SAUSSURE, H.B. de (1779/1786/1796): Voyages dans les Alpes. Précédés d'un Essai sur l'histoire naturelle des Environs de Genève. 4 Bde. Neuchâtel/Genève.

SCHALLER-DONAUER, A. (1925): Zeichensteine in Uri. In: Schweizer Archiv für Volkskunde, Bd. 26, H. 1: 300-301.

SCHINER, H. (1812): Description du département du Simplon ou de la ci-devant République du Valais.

\section{Ungedruckte Quellen}

\section{Chroniken}

CHRONIK VON EISTEN (VS): Zusammengestellt von Br. Stanislaus, Kapuziner. 1978. SA 1227 b.

MICHEL CHRONIK: von Moritz Michel von Ernen (1756-1836). Pfarrarchiv Ernen.

PERRIG CHRONIK (1770): Kantonsarchiv Sitten, AV L 140.

CHRONIQUE DE RIEDMATTEN (1708): Kantonsarchiv Sitten, AV L 152.

HISTORIA COLLEGII SOCIETATIS JESU BRIGAE IN VALLESIA. Geschichte des Kollegiums Brig (1602-1700), handschriftliche Kopie. Kantonsarchiv Sitten, AV L 149.
SCHNEEBELI, W. (1976): 'Untersuchungen von Gletscherschwankungen im Val de Bagnes. In: Die Alpen, 52. Jg., Nr. 3/4: 5-57. Bern.

SCHNIDRIG, A.L. (1952): Grächen. Schweizer Heimatbücher. Bd. 49/50. Verlag Paul Haupt, Bern.

SCHÖNWIESE, C.D. (1979): Klimaschwankungen. SpringerVerlag, Berlin/Heidelberg/New York.

SCHWEINGRUBER, F.H./BRÄKER, O.U./SCHÄR, E. (1979): Dendroclimatic studies on conifers from central Europe and Great Britain. In: Boreas, Vol. 8: 427-452. Oslo.

STUMPF, J. (1547/1548/1586): Gemeiner loblicher Eydgenoschafft Stetten/Landen und Voelckeren Chronick wirdiger thaaten beschreybung... Durch Johann Stumpffen beschrieben. Zürich.

STUMPF, J. (1554): Schwytzer Chronica/Auss der grossen in ein handbüchle zusamen gezogen: in welcher nach der jarzal begriffen ist gemeiner loblicher Eydgenoschafft zeyt... biss auff das jar... 1546... Durch Johann Stumpffen gestellt. Zürich.

TYNDALL, J. (1898): Die Gletscher der Alpen. Autorisierte deutsche Ausgabe. Braunschweig.

VENETZ, I. (1833): Mémoire sur les variations de la température dans les Alpes de la Suisse, rédigé en 1821. Denkschr. der Schweiz. Naturforsch. Ges.

WETTER, W. (1979): Neuzeitliche Gletschergeschichte im Mont Blanc-Massiv. Unteres Vallée de Chamonix. Diplomarbeit, Manuskr. Geogr. Inst. Univ. Zürich.

ZUMBÜHL, H.J. (1978): vgl. PFISTER, Ch. et al. 1978.

ZUMBÜHL, H.J. (1980): Die Schwankungen der Grindelwaldgletscher in den historischen Bild- und Schriftquellen des 12. bis 19. Jahrhunderts. Birkhäuser Verlag, Basel/Boston/Stuttgart.

\section{Dokumente}

Dokumente der Gemeinde Grächen (aufbewahrt im Kantonsarchiv Sitten).

\section{E 1:7. April 1603}

E 2: Abschrift von E 1 aus dem 17./18. Jahrhundert

E 7: 30. Mai 1639

E 12: 28. März 1803

E 12/1, 2: Abschrift, evtl. Neufassung aus dem Jahre 1849.

E 12/3: Inhalt identisch mit E 12/1, 2 mit einigen geschichtlichen Zusätzen. 